\title{
Pulsed Femtosecond-Laser Treatment and Deep Reactive Ion Etching of Diamond
}

\author{
Thomas Wulz, ${ }^{1}$ Brian K. Canfield, ${ }^{2}$ Lloyd M. Davis, ${ }^{2,3}$ Stefan Spanier, ${ }^{3}$ and Eric Lukosi ${ }^{1}$
}

Keywords: Diamond; Femtosecond laser machining; Raman; Carbon; Reactive-ion etching; Through-diamond vias.

\begin{abstract}
This paper reports the deep reactive ion etching of femtosecond laser machined highaspect graphitic channels through diamond. To begin, two chemical vapor deposition-grown diamond plates, one single-crystal and the other polycrystalline, are processed using the bulk microstructural modification technique of femtosecond-pulsed laser machining. Multiple laser parameters are varied in processing the polycrystalline sample, including laser pulse frequency, pulse energy, and number of pulses per micron step size between the back and the front of the diamond, whereas a constrained set of parameters is used with the single-crystal sample. Over the range of parameters evaluated for the polycrystalline diamond, Raman analysis exhibits photoluminescence from defects created near the surface through the laser machining process, but little, if any, graphite-like features. However, a Raman signal from graphite-like material is observed on the surface of the graphitic channels for the single-crystal diamond, from which it is determined that greater diamond to graphite-like phase conversion is obtained with a larger number of pulses per translation step and with a higher laser pulse energy. Further, a larger number of pulses per step results in strong luminescence, observed during Raman investigation. Subsequent deep reactive ion etching of the samples reveals that the laser-treated channels etch considerably faster near the surface than the surrounding diamond, and that a high relative etch rate is obtained even when graphite-like features are absent from the Raman spectra. The highest relative etch rate of the single-crystal sample was $1.61 \mu \mathrm{m} / \mathrm{min}$.
\end{abstract}




\section{Introduction}

Diamond is a well-studied material, with many favorable attributes for numerous applications [15]. In reference to the use of lasers on diamond substrates, the applications have included surface treatments [6-8], growth and ablation/etching of diamond [9-11], diffraction gratings and other elements for high power optical systems [12-14], and graphitic/conductive channel formation [8, 15-21]. The potential for directly writing conductive channels onto or into diamond for electronic applications is appealing, given the potential speed and high-dielectric constant of diamond, which are utilized in applications such as high-power switches [4]. Recent investigations, however, have demonstrated that the channel conductivity is dependent on the laser parameters $[5,20,22,23]$. When femtosecond (fs) lasers are used, the scan speed of the laser focus through the substrate during the creation of graphitic channels is found to be a key parameter in determining the resistivity of the channels. In one recent report, a maximum conductivity of 64 $\mathrm{S} / \mathrm{m}$ was obtained with a scan rate of $20 \mu \mathrm{m} / \mathrm{s}$ [22]. The result was correlated to the maximum ratio of the $\mathrm{G}\left(1500 \sim 1630 \mathrm{~cm}^{-1}\right)$ peak to the $\mathrm{D}$ peak $\left(1350 \mathrm{~cm}^{-1}\right)$ in the Raman spectrum, where this ratio increases with increasing disorder. These authors also find that merely increasing the laser intensity results in non-uniform creation of graphitic centers, which they attribute to the Kerr effect (nonlinear optical self-focusing of the laser). However, when nanosecond (ns) lasers are used, the conductivity of the channels increases by an order of magnitude [23], but when used for the fabrication of 3D diamond detector devices, the diamond exhibits reduced charge collection efficiency and/or polarization (a competing internal electric field due to charge trapping) [20]. In any case, the conductivity obtained with a ns-laser is still an order of magnitude below that of crystalline graphite, and 1000 times lower than that of compressed graphite powder.

It has been indicated that for ns lasers the thermalization time of excited electrons plays a role in determining the created structure, whereas fs lasers do not allow the electrons to thermalize with the lattice during the delivery of the pulses [8]. For processing that allows thermalization with the lattice to take place, the heating of the surrounding material and the density change between diamond and graphitic-like carbon can result in mechanical stresses and cracking, and the residual pressure can be observed through Raman investigation of the stress-induced shift in the G peak [5]. Further, internal pressure can slow the progression of graphitization, even reversing it and creating nanodiamond crystallites [5, 18].

The results to date on the conductivity of graphitic channels created by either ns or fs laser machining through bulk diamond indicate that it will be very challenging to produce lowresistance channels, such as those required for fast, high-power switches or other devices. However, as demonstrated in this paper, there is the possibility to laser machine throughdiamond channels that are partially graphitized, which can be etched away at a significantly faster rate than the untreated diamond to thereby create through-diamond vias (TDVs). The TDVs may be subsequently filled with a conductive material, as is commonly done in the silicon industry $[24,25]$. One report has used a boron-doped layer on silicon and laser treatment to create a hard mask for deep reactive ion etching, but did not use a diamond laser thicker than 1.5 $\mu \mathrm{m}$ [26]. This paper reports the effects of various fs pulsed laser processing conditions on the level of graphitization of two chemical vapor deposition (CVD)-grown diamonds, one singlecrystal (scCVD) and the other polycrystalline (pcCVD), and the deep reactive ion etching 
(DRIE) of the associated laser-created non-diamond carbon phase regions. DRIE, which is a form of dry chemical etching, can selectively remove $\mathrm{sp}^{2}$ bonded over $\mathrm{sp}^{3}$ bonded carbon to create TDVs through the diamond substrate with dimensions defined by the laser treatment process.

\section{Materials and Methods}

The laser system used in this investigation consists of a Ti:S oscillator (Spectra-Physics, Tsunami) with a regenerative amplifier (Coherent, RegA 9000), which provides a pulse duration of $\sim 130$ fs at $797 \pm 8 \mathrm{~nm}$ with a maximum energy of $4 \mu \mathrm{J}$. Very tight aberration-corrected focusing of the beam into the diamond is accomplished using a beam expander made of three lenses together with an aspheric lens with numerical aperture (in air) of 0.68 . The beam expander is designed to deliberately introduce spherical and chromatic aberration, and two of its lenses are repositioned by motorized translators using a look-up table of lens positions to adjust the aberration so that it pre-compensates for that produced by refraction of the beam at the surface of the diamond. As theoretically determined using optical design software (Zemax OpticStudio 15), the aberration pre-compensation enables a focal spot diameter of $\sim 1 \mu \mathrm{m}$ to be retained as the depth of focus is translated through the diamond. During laser processing of each test channel, the initiation of a structural change within the diamond is monitored by observing diffraction of the beam from a green laser pointer, which is collinear with the fs laser beam, and following laser processing, the samples are inspected using an optical microscope. The aberration compensation when focusing through the maximum depth of diamond can be experimentally verified by creating a feature at the rear surface of size $\sim 1 \mu \mathrm{m}$ using a single pulse with energy just above threshold. However, for the operating conditions presented below, the resulting channel diameters are typically larger and depend on the number and energy of laser pulses used.

In our studies, two diamond substrates are treated in ambient air using the fs-pulsed, aberrationcorrected laser focus: Sample 1, a $500 \mu \mathrm{m}$ thick, pcCVD diamond, and sample 2, a $300 \mu \mathrm{m}$ thick, scCVD diamond. Sample 1 is investigated with a wide variation of the laser parameters, including pulse energy, E $(0.2-4 \mu \mathrm{J})$, pulse frequency $(0.033-250 \mathrm{kHz})$, number of pulses per position, $\mathrm{N}(2-5000)$, discrete differential step height between laser treatment positions, $\Delta \mathrm{z}(2-$ $12 \mu \mathrm{m})$, as well as continuous translation $(10-100 \mu \mathrm{m} / \mathrm{s})$.

Sample 2 is treated using a more constrained range of experimental parameters, guided by the results from sample 1. Two different sets of parameters are investigated, each identified by a "1" or " 2 " ablated into the diamond surface above the array of test points. For both arrays (4 rows $x$ 4 columns), the laser pulsing frequency is kept constant at $2 \mathrm{kHz}$, and the step size is $\Delta \mathrm{z}=2 \mu \mathrm{m}$. Array 1 uses 10 laser pulses per z-step, while array 2 uses 1000 pulses per step. In each of the arrays, the laser energies are $\mathrm{E}=0.4,0.8,1.2$, and $1.6 \mu \mathrm{J}$ from the top row to the bottom row, while the parameters are the same in each column of each array. Figure 1 (a) and (b) show optical microscope images of the front surfaces of samples 1 and 2 . In each case, the lateral shift between test points, obtained with a manual micrometer stage, is $\sim 100 \mu \mathrm{m}$. The laser-machined channel at each test point is continuous between the front and back of the diamond, as may be seen in Figure 1 (c), which presents a set of images of 6 channels in pcCVD diamond captured with a long working distance, low numerical aperture objective, with focus adjusted to the front, middle, and back, and with the sample tilted so as to see a side view of the channels. 


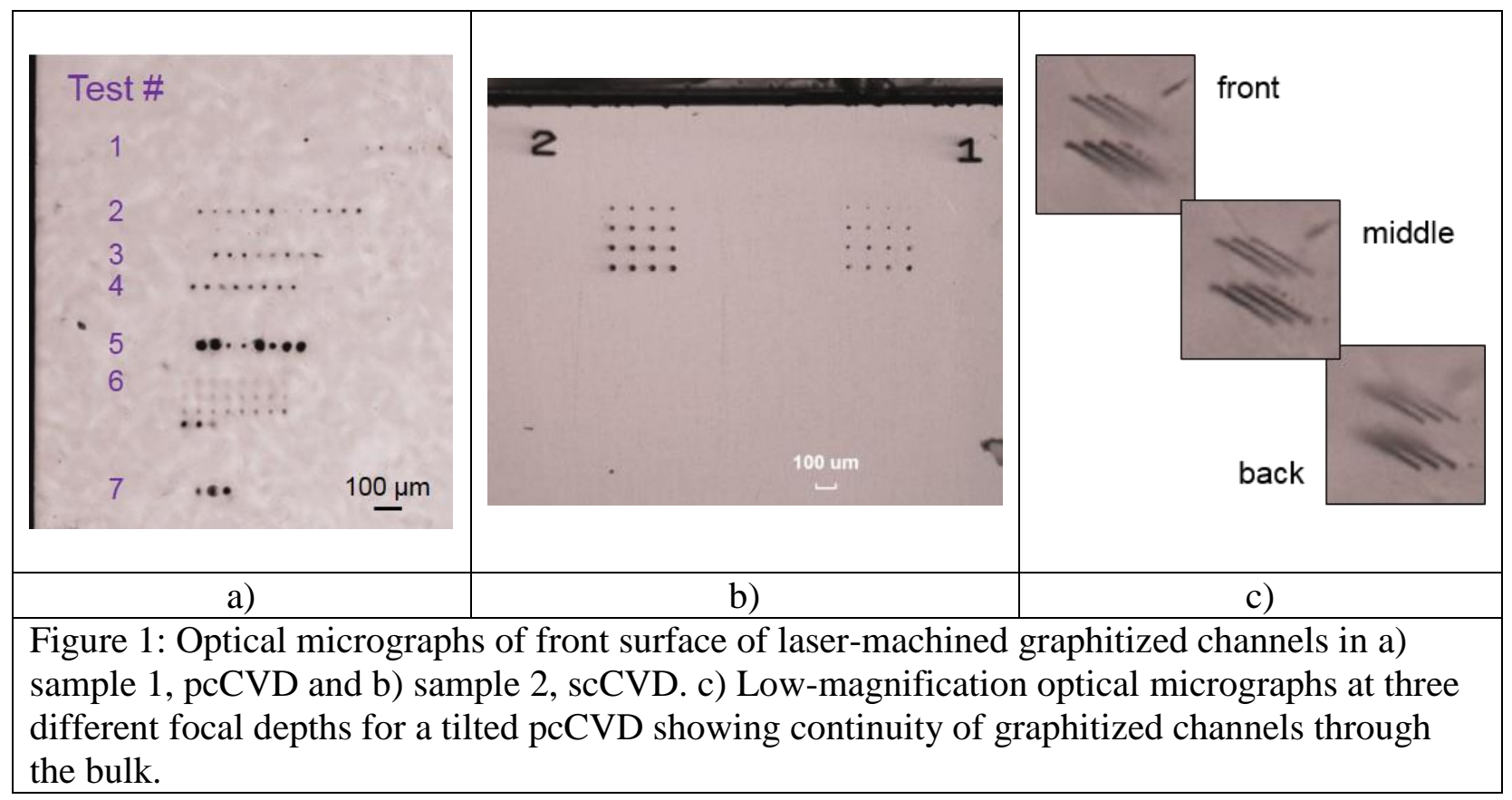

Raman spectral measurements on the created graphitic channels are acquired using a Renishaw inVia confocal Raman microscope utilizing a $633 \mathrm{~nm}$ excitation source with a $1 \mu \mathrm{m}$ diameter spot size and grating with 1200 lines/mm, well within the diameter of column diameters created during laser treatment. Correlation between the laser processing parameters and surface Raman peak intensities of diamond and laser-induced spectral effects on the top surface of each column were determined. Measurement of the resistance of the created channels would be an alternate means of characterizing the degree of graphitization of the laser-machined features, but was not performed in this study due to difficulties in forming reliable electrical contacts with the ends of the columns.

DRIE of the laser-treated sample 2 is carried out in an Oxford Instruments Plasmalab100 machine capable of independently controlling the inductively-coupled plasma (ICP) coil power and radio frequency (RF) table power. During the etching process, the diamond was mounted to a four-inch silicon carrier wafer using thermally conductive Krytox ${ }^{\mathrm{TM}}$ vacuum oil. The dry chemical etching of sample 2 is conducted with oxygen, an ICP coil power of $2.5 \mathrm{~kW}$, and RF table power of $150 \mathrm{~W}$. The total etch depth of each graphitized channel was kept below $20 \mu \mathrm{m}$ to reduce systematic effects from mass transport limitations in channels of differing diameter. The surface topology of the laser processed and etched regions was characterized via sputtering chromium on the surface of the diamond following by optical profilometry using a Veeco $8800 \mathrm{NT}$ white light interferometer in vertical scanning interferometry mode.

\section{Results and Discussion}

In laser machining each test channel, a structural change within the diamond is apparent from the diffraction of the collinear green laser pointer beam. However, for sample 1, it is found that, for low pulse energies $(\mathrm{E})$ and number of pulses $(\mathrm{N})$, features at the front surface are barely visible in the microscope image, as may be seen in Figure 2. On the other hand, for the highest pulse energy $(4 \mu \mathrm{J})$, a crack extending from the channel sometimes appears at the front surface. For 
some conditions, the channels appear markedly different on the front and back of the sample. Also, the direction of translation is found to have little effect on the resultant appearance of the graphitized channels, perhaps because much of the energy of each pulse converges at high angles rather than propagating along the axis and being obstructed by previously formed graphitization. Furthermore, as shown in Figure 3, for the highest pulse energy, ablation, or cratering, sometimes appears on the front face of the sample The extent of cratering and asymmetry of the observed features on the front and back of the sample increase as the number of laser pulses is increased. Also, the appearance of the laser-treated region of sample 1 on the back surface seems less affected by changes in the number of pulses than that at the front. Overall, in both samples, correlations are observed between the apparent sizes of the graphitized channels at the front and back and the laser pulse energy and number of pulses.
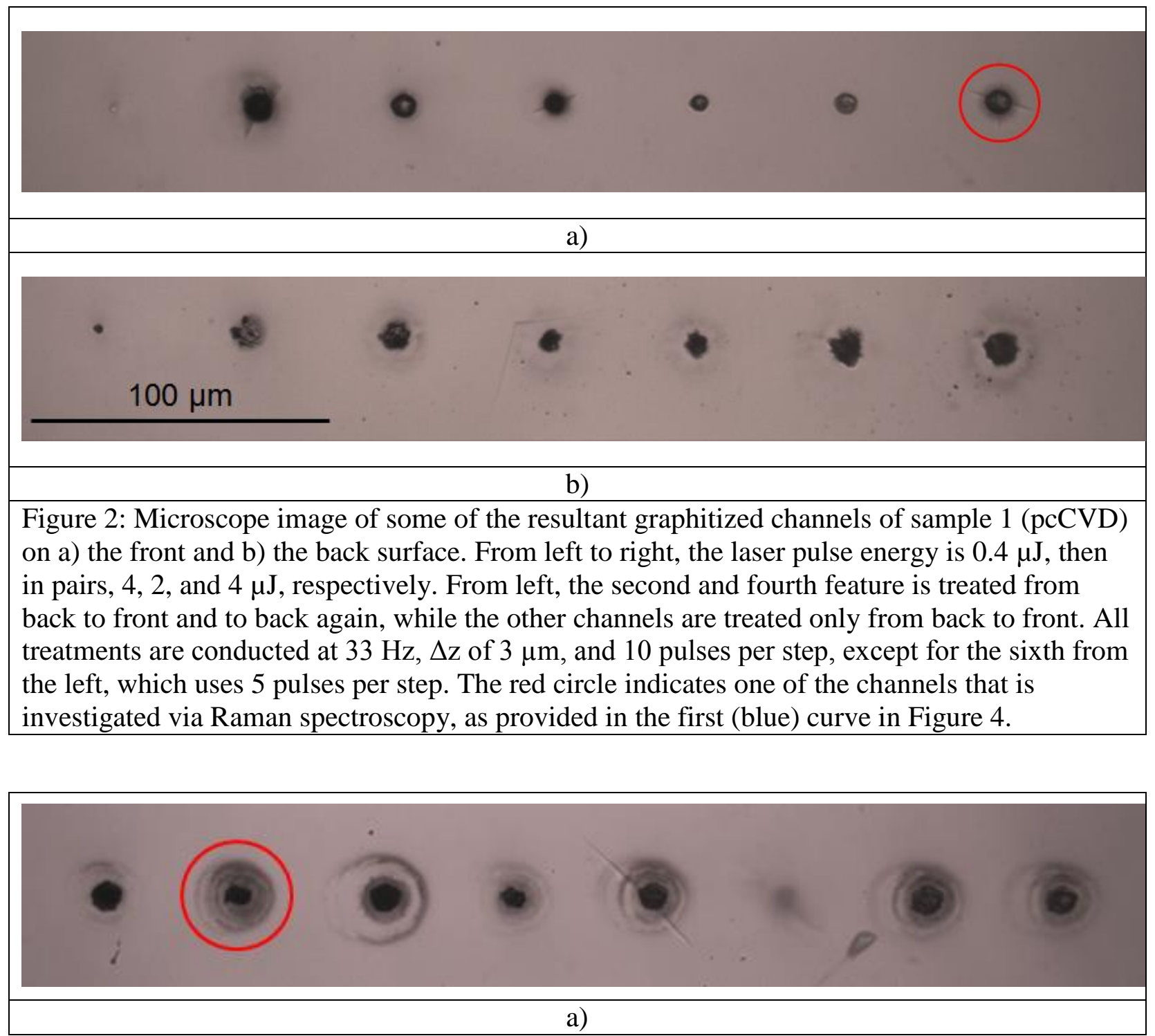


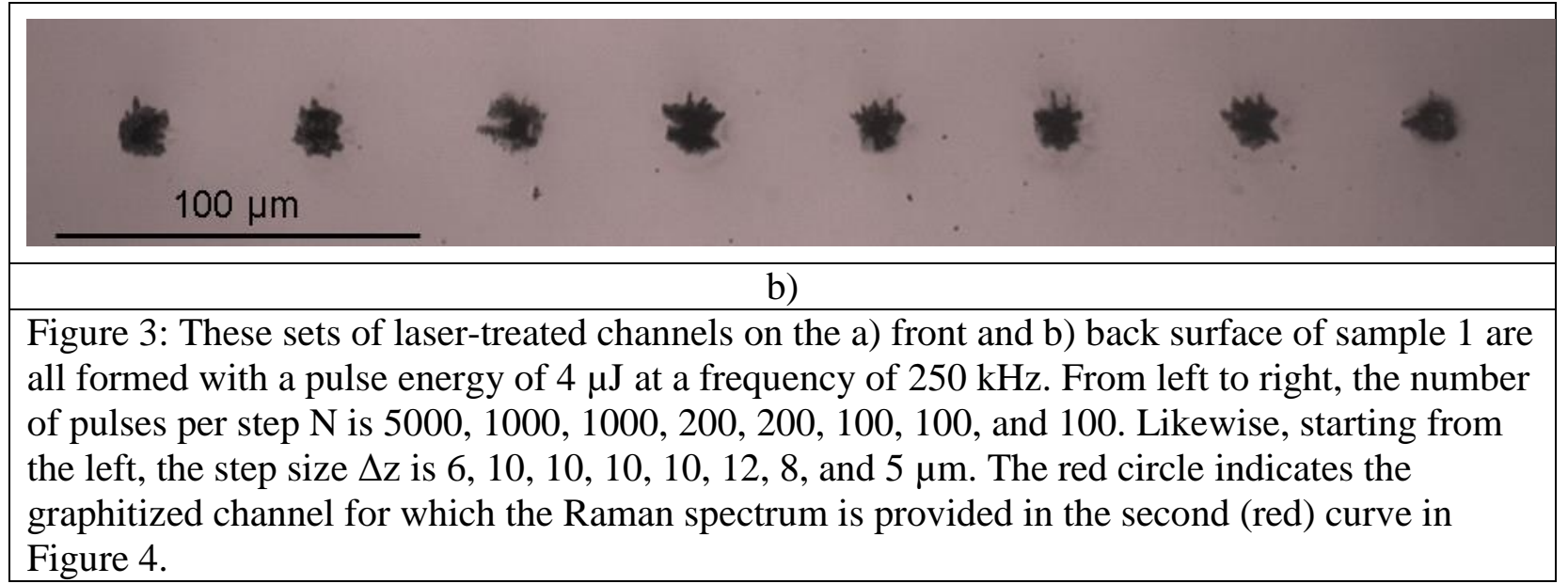

For sample 1, the wider range of laser processing parameters used leads to a correspondingly more different set of Raman spectra. Sample 2 (scCVD), which is machined with a more constrained range of parameters based on results from sample 1, is found to yield a more consistent set of graphitized channels, both visually in the microscope and through Raman investigation. For sample 1, Figure 4 displays the surface Raman spectra of the two laser-treated channels that are identified by red circles in Figures 2 and 3. In the Raman spectrum of the identified channel in Figure 2, the characteristic $1332 \mathrm{~cm}^{-1}$ Raman peak for $\mathrm{sp}^{3}$ bonded carbon is clearly apparent, and there is also an increase in Raman counts around $2250 \mathrm{~cm}^{-1}$. For the Raman spectrum of the channel in Figure 3, there is a distinct, strong peak around $2350 \mathrm{~cm}^{-1}$, followed by an even more intense and broader feature at $3080 \mathrm{~cm}^{-1}$. The difference between the two Raman spectra is due to the larger number of pulses and higher pulsation frequency used for the column in Figure 3, and the intense Raman features are attributed to photoluminescence from defects created through the laser machining process [27]. Visually, the laser-treated channel from Figure 3 is roughly circular on the front face with noticeable cratering and peripheral rings, while the back is dark black and similar in shape to an amoeba or 7-point star. However, neither the $1355 \mathrm{~cm}^{-1}$ disordered graphite nor the $1580 \mathrm{~cm}^{-1}$ single-phonon, crystalline graphite Raman peaks are seen in any of the Raman spectra from sample 1. We suspect that the lack of graphitized scattering peak from the column identified in Figure 2 is a result of the low laser pulse frequency and $\Delta \mathrm{z}$. The Raman results for the column identified in Figure 3 used a much higher laser pulse frequency, but the results suggest that the induced damage in the pcCVD diamond resulted in overwhelming luminescent-type defects in comparison to the scattering signal at either $1355 \mathrm{~cm}^{-1}$ and $1580 \mathrm{~cm}^{-1}$. 


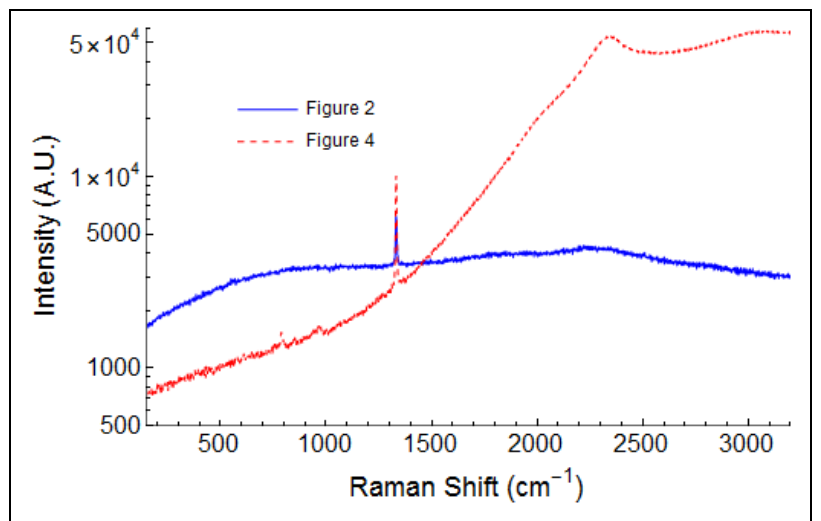

Figure 4: Raman spectra of two treated regions of sample 1 . The laser pulse energy, frequency, $\Delta z$, and number of pulses per step $\mathrm{N}$ for each spectrum is $(0.4 \mu \mathrm{J}, 33 \mathrm{~Hz}, 3 \mu \mathrm{m}$, and 10 , solid blue curve), and $(4 \mu \mathrm{J}, 250$ $\mathrm{kHz}, 10 \mu \mathrm{m}$, and 1000, dashed red curve).

Surface Raman spectra of laser-treated channels in column 1 of both arrays 1 and 2 and for rows 1 through 4 are provided in Figure 5. The Raman spectra are quite consistent across the four laser-treated channels of each row in the array, exhibiting both the diamond zero-phonon line around $1332 \mathrm{~cm}^{-1}$ and a broad distribution extending from the zero-phonon line to $\sim 1600 \mathrm{~cm}^{-1}$. The crystalline $1580 \mathrm{~cm}^{-1}$ peak is apparent in the spectra, which suggests that the channels partially consist of crystalline graphite, but also contain $\mathrm{sp}^{2}$ bonded defects. Furthermore, in array 1, a small feature around $1090 \mathrm{~cm}^{-1}$ is observed for rows 2 through 4 , which is attributed to diamond nanocrystals or to Z-carbon in [21]. In array 2, a large luminescence continuum is observed, starting from $\sim 1700 \mathrm{~cm}^{-1}$.

The ratio of diamond Raman peak intensity to the graphite-like region, summed between 1570 $\mathrm{cm}^{-1}$ and $1590 \mathrm{~cm}^{-1}$, for sample 2 is provided in Figure 6. Each data point is the average of the observed ratios for the four laser-treated channels after subtraction of the luminescent background, and the error bars represent the standard deviation. A noticeable correlation of the diamond peak to graphite-like ratio with laser energy is observed, where array 2 portrays a larger increase in the ratio with laser pulse energy than array 1 . The relationship between the number of pulses, N, per $\Delta z$ (10 for array 1 and 1000 for array 2) and laser energy suggests that increasing the laser energy for higher $\mathrm{N}$ increases the rate of graphitization faster than using a lower $\mathrm{N}$ per $\Delta \mathrm{z}$, although the rate of graphitization is higher for lower $\mathrm{N}$ in array 1 than higher $\mathrm{N}$ in array 2 for the lowest two laser pulse energies. 


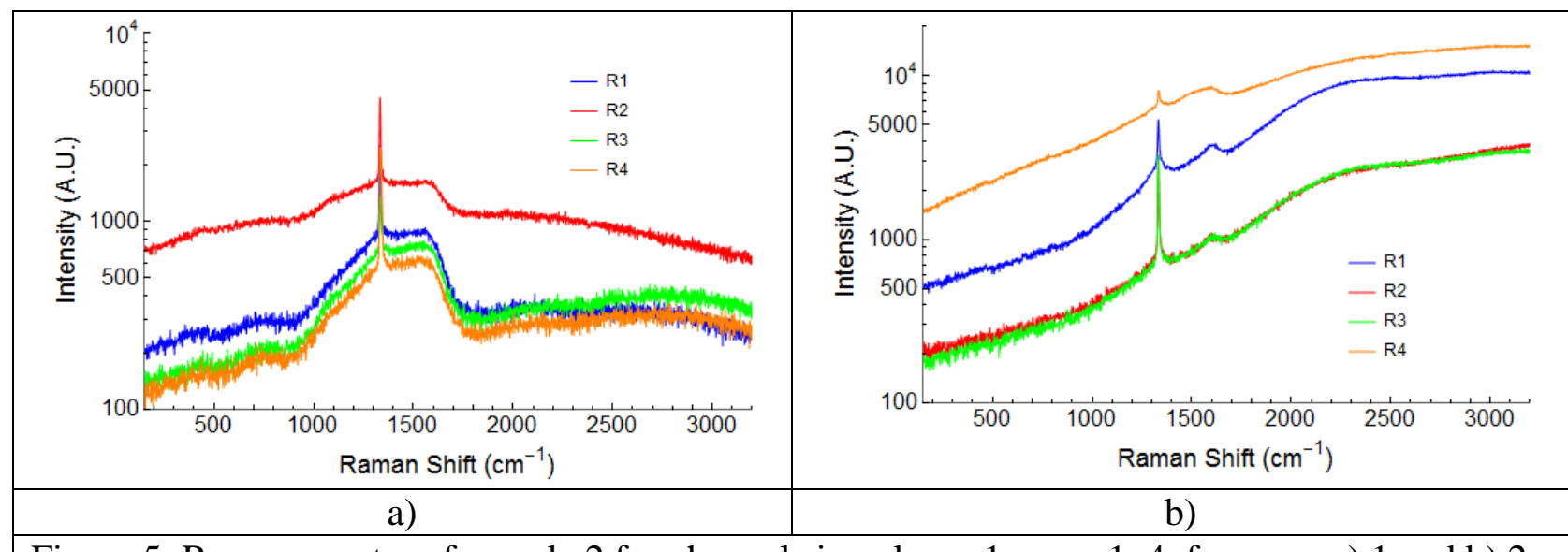

Figure 5: Raman spectra of sample 2 for channels in column 1, rows $1-4$, for array a) 1 and b) 2 .

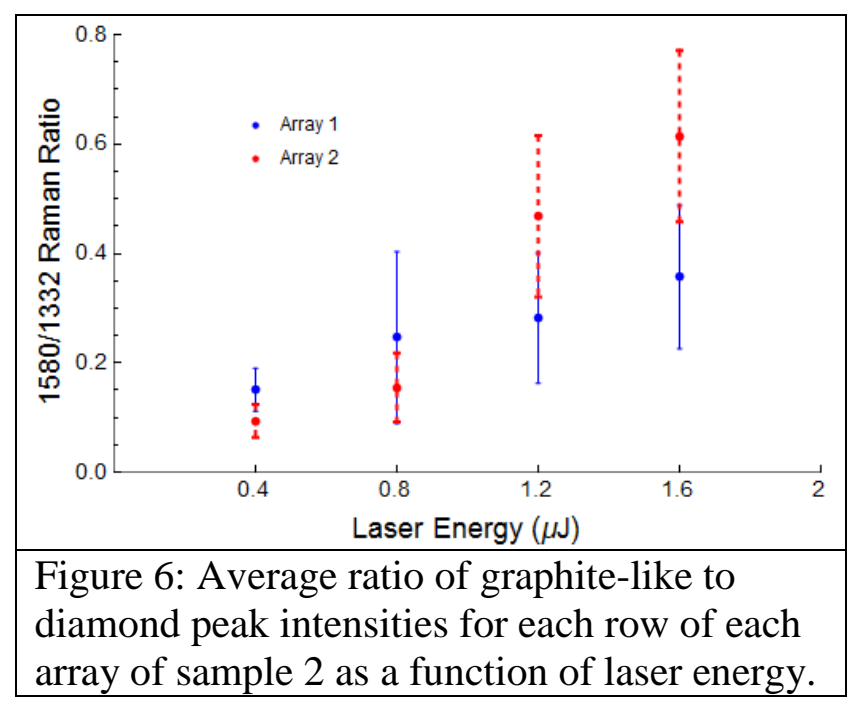

DRIE etching of sample 1 demonstrated that the etch rate of the graphitic channels compared to the bulk diamond substrate was faster, measured through contact profilometry, but white light interferometry was unsuccessful due to the non-reflecting nature of the columns, and the sample was inadvertently destroyed before additional investigations could take place. Results from DRIE of sample 2 consistently shows that the laser-treated channels etch considerably faster than the surrounding diamond. The averaged etch rates from the collected data for each row in each array of sample two is provided in Figure 7. As shown, the average relative etch rate increases as a function of laser energy. The highest relative etch rates were $1.61 \pm 0.02 \mu \mathrm{m} / \mathrm{min}$ for array 1 , and $1.16 \pm 0.16 \mu \mathrm{m} / \mathrm{min}$ for array 2 . Interestingly the channels of array 1 etch faster than those of array 2 , even though array 1 uses 10 laser pulses per $\Delta z$, while array 2 uses 1000 pulses per $\Delta z$. The etch rate of array 1 increases rapidly with the first increase in laser pulse energy, but then tapers off between the $1.2 \mu \mathrm{J}$ and $1.6 \mu \mathrm{J}$. Array 2, on the other hand, exhibits a rapid increase in etch rate between the first two and last two laser pulse energies, which is consistent with the diamond-like to diamond Raman peak ratio data provided in Figure 6. The apparent difference in etch rates between the two arrays suggest that a lower number of pulses is more effective for etching, but higher laser pulse energies, creating the observed feature around $1090 \mathrm{~cm}^{-1}$, may reduce etch rates. It is unclear with the data at-hand whether the 1000 laser pulses per $\Delta z$ would 
surpass the etch rates when 10 laser pulses per $\Delta \mathrm{z}$ are used for laser pulse energies greater than $1.6 \mu \mathrm{J}$, but the trends observed in Figures 6 and 7 suggest that this may be the case.

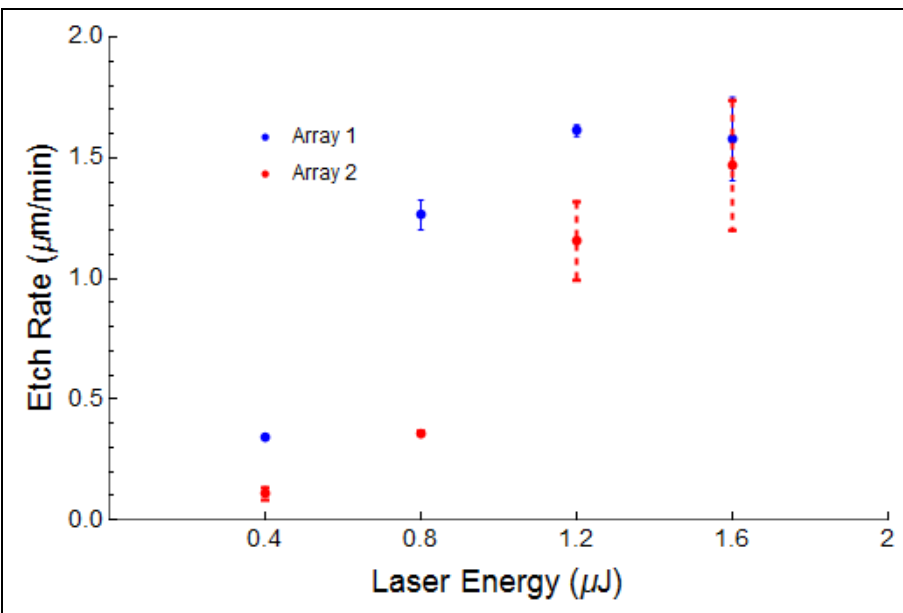

Figure 7: Average DRIE etch rate of the surface of each laser-treated column for both arrays of sample 2 (scCVD) as a function of laser pulse energy. The error bars are the standard deviations and do not account for systematic errors from the profilometry hole-depth estimates.

\section{Conclusions}

Two chemical vapor deposition-grown diamond plates, one single-crystal and the other polycrystalline, are processed using the bulk microstructural modification technique of femtosecond-pulsed laser machining. Microscope images and confocal Raman spectra of the surface of processed regions reveal varying degrees of phase transformation from $\mathrm{sp}^{3}$ bonded carbon to $\mathrm{sp}^{2}$ bonded and disordered $\mathrm{sp}^{2}$ bonded carbon, as ascertained by the appearance of dark graphite-looking carbon in the images and the presence in the spectra of the characteristic 1332 $\mathrm{cm}^{-1}$ diamond peak and a broad distribution of features from the zero-phonon line to $\sim 1600 \mathrm{~cm}^{-1}$. Many laser parameters investigated with the pcCVD diamond did not result in graphite-like peaks in the spectra but did yield an increased luminescence continuum starting from $\sim 1700 \mathrm{~cm}^{-}$ ${ }^{1}$, although graphite-like discoloration is observed in the microscope images.

For sample 2, a general trend between the laser treatment energy and the surface graphite-like to diamond Raman peak intensity ratio was observed. It was found that a lower number of laser pulses per $\Delta \mathrm{z}$ resulted in a larger ratio, but also exhibited a small feature around $1090 \mathrm{~cm}^{-1}$, attributed to nanocrystalline diamond or Z-carbon [21]. However, the polycrystalline nature of sample 1 suggests that a higher rate of conversion from $\mathrm{sp}^{3}$ to $\mathrm{sp}^{2}$ bonded carbon should occur for the same processing parameters as sample 2 but was not observed.

During deep reactive ion etching (DRIE) of the top $\sim 20 \mu \mathrm{m}$ of the laser-machined graphitized channels, a general trend was not observed for the laser processing parameters for sample 2 . It was observed that, for 1000 pulses per $\Delta z$, the etch rate increased significantly for laser pulse 
energies surpassing $1 \mu \mathrm{J}$, consistent with the channel surface diamond-like to diamond Raman peak intensities. However, for 10 pulses per $\Delta z$, the etch rate increased dramatically for an increase in laser pulse energy between $0.4 \mu \mathrm{J}$ and $0.8 \mu \mathrm{J}$, but then tapered off for higher laser pulse energies. The observed feature in the Raman spectra of array 1 for sample 2 around 1090 $\mathrm{cm}^{-1}$ may be indicative of the reduced etch rate for high laser pulse energy. The luminescence observed in array 2 of sample 2 indicates that the larger 1000 laser pulses per $\Delta z$, while increasing the surface diamond-like to diamond Raman peak intensities, may also be etched at a faster rate than the carbon structures created using 10 laser pulses per $\Delta z$. Still, the reader should be aware that these results are for etching near-surface laser-machined graphitic columns, and the etch properties may be different deeper into the bulk of the columns. However, the observed relative etch rate of the laser-machined graphitic columns in the single-crystal diamond substrate is several times faster than bulk single crystal diamond, which etches at a rate of no more than a few hundred nanometers per minute [28-31]. Therefore, with a suitable hard mask to protect the diamond substrate, etch rates of laser-treated columns may be as high as $2 \mu \mathrm{m} / \mathrm{min}$, sufficient to create TDVs in a reasonable timeframe for a variety of applications, provided that the etching rates do not deviate significantly from the observed rates near the surface.

\section{Acknowledgements}

The diamond etching and Raman spectroscopy reported in this research was conducted at the Center for Nanophase Materials Sciences, which is a DOE Office of Science User Facility. 


\section{References}

1. Balmer, R.S., Brandon, J.R., Clewes, S.L., Dhillon, H.K., Dodson, J.M., Friel, I., Inglis, P.N., Madgwick, T.D., Markham, M.L., Mollart, T.P., Perkins, N., Scarsbrook, G.A., Twitchen, D.J., Whitehead, A.J., Wilman, J.J., and Woollard, S.M., Chemical vapour deposition synthetic diamond: materials, technology and applications. J. Phys: Condens. Matter, 2009. 21.

2. Balmer, R.S., Friel, I., Woollard, S.M., Wort, C.J.H., Scarsbrook, G.A., Coe, S.E., ElHajj, H., Kaiser, A., Denisenko, A., Kohn, E., and Isberg, J., Unlocking diamond's potential as an electronic material. Philosophical Transactions of the Royal Society A: Mathematical, Physical and Engineering Sciences, 2008. 366(1863): p. 251-265.

3. Prelas, M.A., Popovici, G., and Bigelow, L.K., Handbook of Industrial Diamonds and Diamond Films. 1998, New York: Marcel Dekker.

4. Sussmann, R.S., CVD Diamond for Electronic Devices and Sensors. 2009, West Sussex, U.K.: John Wiley \& Sons.

5. Konov, V.I., Laser in micro and nanoprocessing of diamond materials. Laser \& Photonics Reviews, 2012. 6(6): p. 739-766.

6. Pimenov, S.M., Kononenko, V.V., Ralchenko, V.G., Konov, V.I., Gloor, S., Lüthy, W., Weber, H.P., and Khomich, A.V., Laser polishing of diamond plates. Applied Physics A, 1999. 69(1): p. 81-88.

7. Wu, Q., Ma, Y., Fang, R., Liao, Y., Yu, Q., Chen, X., and Wang, K., Femtosecond laserinduced periodic surface structure on diamond film. Applied Physics Letters, 2003.

82(11): p. 1703-1705.

8. Wang, C.Z., Ho, K.M., Shirk, M.D., and Molian, P.A., Laser-induced graphitization on a diamond (111) surface. Physical Review Letters, 2000. 85(19): p. 4092-4095.

9. Preuss, S. and Stuke, M., Subpicosecond ultraviolet laser ablation of diamond: Nonlinear properties at $248 \mathrm{~nm}$ and time-resolved characterization of ablation dynamics. Applied Physics Letters, 1995. 67(3): p. 338-340.

10. Rothschild, M., Arnone, C., and Ehrlich, D.J., Excimer-laser etching of diamond and hard carbon films by direct writing and optical projection. Journal of Vacuum Science \& Technology B, 1986. 4(1): p. 310-314.

11. Rebello, J.H.D., Straub, D.L., and Subramaniam, V.V., Diamond growth from a CO/CH4 mixture by laser excitation of CO: Laser excited chemical vapor deposition. Journal of Applied Physics, 1992. 72(3): p. 1133-1136.

12. Kononenko, V.V., Konov, V.I., Pimenov, S.M., Prokhorov, A.M., Pavelyev, V.S., Soifer, V.A., Luedge, B., and Duparre, M.R., Laser shaping of diamond for IR diffractive optical elements. Proc. SPIE 4426, Second International Symposium on Laser Precision Microfabrication, 2002. 4426(128): p. 128-134.

13. Pavelyev, V., Soifer, V., Konov, V., Kononenko, V., and Volkov, A., Diffractive Microoptics for Technological IR-Lasers. High-Power and Femtosecond Lasers:

Properties, Materials and Applications.-Ed. by Paul-Henri Barret and Michael Palmer.Nova Science Publishers, Inc, 2009: p. 125-158.

14. Pavelyev, V.S., Soifer, V.A., Golovashkin, D.L., Kononenko, V.V., Konov, V.I., Pimenov, S.M., Duparre, M.R., and Luedge, B., Diamond DOEs for focusing IR laser beams into pregiven focal domains. Proc. SPIE 5182, Wave-Optical Systems Engineering II, 2004. 5182(222): p. 222-232. 
15. Kononenko, T.V., Komlenok, M.S., Pashinin, V.P., Pimenov, S.M., Konov, V.I., Neff, M., Romano, V., and Lüthy, W., Femtosecond laser microstructuring in the bulk of diamond. Diamond and Related Materials, 2009. 18(2-3): p. 196-199.

16. Kononenko, T., Ralchenko, V., Bolshakov, A., Konov, V., Allegrini, P., Pacilli, M., Conte, G., and Spiriti, E., All-carbon detector with buried graphite pillars in CVD diamond. Applied Physics A, 2014. 114(2): p. 297-300.

17. Neff, M., Kononenko, T.V., Pimenov, S.M., Romano, V., Lüthy, W., and Konov, V.I., Femtosecond laser writing of buried graphitic structures in bulk diamond. Applied Physics A, 2009. 97(3): p. 543-547.

18. Strekalov, V.N., Konov, V.I., Kononenko, V.V., and Pimenov, S.M., Early stages of laser graphitization of diamond. Applied Physics A, 2003. 76(4): p. 603-607.

19. Lagomarsino, S., Parrini, G., Sciortino, S., Bellini, M., Corsi, C., Gorelli, F., and Santoro, M. Pulsed Laser Fabrication of 3D Diamond Detectors. in RD13 - 11th International Conference on Large Scale Applications and Radiation Hardness of Semiconductor Detectors. 2013. Florence, Italy.

20. Lagomarsino, S., Bellini, M., Corsi, C., Gorelli, F., Parrini, G., Santoro, M., and Sciortino, S., Three-dimensional diamond detectors: Charge collection efficiency of graphitic electrodes. Applied Physics Letters, 2013. 103(23).

21. Lagomarsino, S., Bellinic, M., Corsic, C., Fanettic, S., Gorellic, F., Liontosc, I., Parrinia, G., Santoroc, M., and Sciortinoa, S., Electrical and Raman-imaging characterization of laser-made electrodes for 3D diamond detectors. Diamond and Related Materials, 2014. 43: p. 23-28.

22. Shimizu, M., Shimotsuma, Y., Sakakura, M., Yuasa, T., Homma, H., Minowa, Y., Tanaka, K., Miura, K., and Hirao, K., Periodic metallo-dielectric structure in diamond. Optics Express, 2009. 17(1): p. 46-54.

23. Oh, A., Caylar, B., Pomorski, M., and Wengler, T., A novel detector with graphitic electrodes in CVD diamond. Diamond and Related Materials, 2013. 38(0): p. 9-13.

24. William, S., Semiconductive wafer and method of making the same. 1962, Google Patents.

25. Smith, M.G. and Emanuel, S., Methods of making thru-connections in semiconductor wafers. 1967, Google Patents.

26. Baehr, M., Klein, T., Taeschner, R., and Ortlepp, T. Making use of synthetic diamond layers for $3 d$ micro- and nanoelectronic applications. in Micro-Nano-Integration; 6. GMM-Workshop. 2016.

27. Filik, J., Raman spectroscopy: a simple, non-destructive way to characterise diamond and diamond-like materials. Spectroscopy Europe, 2005. 17(5): p. 10.

28. Ding, G.F., Mao, H.P., Cai, Y.L., Zhang, Y.H., Yao, X., and Zhao, X.L., Micromachining of CVD diamond by RIE for MEMS applications. Diamond and Related Materials, 2005. 14(9): p. 1543-1548.

29. Forsberg, P. and Karlsson, M., High aspect ratio optical gratings in diamond. Diamond and Related Materials, 2013. 34(0): p. 19-24.

30. Hausmann, B.J.M., Khan, M., Zhang, Y., Babinec, T.M., Martinick, K., McCutcheon, M., Hemmer, P.R., and Lončar, M., Fabrication of diamond nanowires for quantum information processing applications. Diamond and Related Materials, 2010. 19(5-6): p. 621-629. 
31. Otterbach, R. and Hilleringmann, U., Reactive ion etching of CVD-diamond for piezoresistive pressure sensors. Diamond and Related Materials, 2002. 11(3-6): p. 841844. 
Graphical abstract

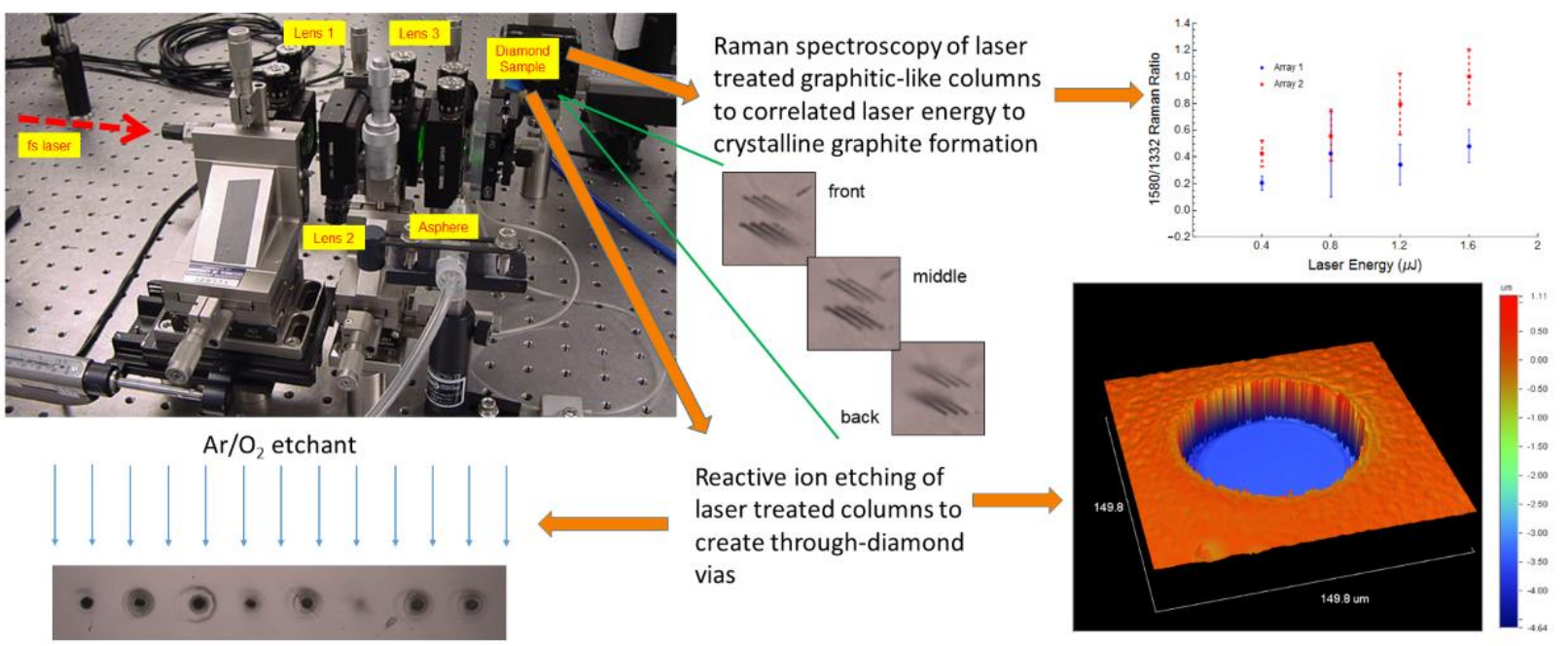

